\title{
Self-administered medical abortion pills: evaluation of the clinical outcome and complications among women presenting with unsupervised pill intake to a tertiary care hospital: a cross-sectional study
}

\author{
Shreya Singh, Sheela Shivamonga Rangappa*
}

Department of Obstetrics and Gynaecology, Sri Deveraj Medical College, Tamaka, Kolar, Karnataka, India

Received: 15 June 2021

Revised: 03 August 2021

Accepted: 04 August 2021

\section{*Correspondence:}

Dr. Sheela Shivamonga Rangappa,

E-mail: drsheela@gmail.com

Copyright: (C) the author(s), publisher and licensee Medip Academy. This is an open-access article distributed under the terms of the Creative Commons Attribution Non-Commercial License, which permits unrestricted non-commercial use, distribution, and reproduction in any medium, provided the original work is properly cited.

\section{ABSTRACT}

Background: In India, it is becoming a public health problem due to easy over-the-counter abortion pill availability despite the legal ban, widespread misuse by non-allopath doctors, dais and quacks, and ignorance on part of women. The pills are being dispensed blindly without proper medical evaluation and even without ruling out the contraindications. In India, abortion facility is available legally under the MTP act, 1971.

Methods: Its cross-section study including 81 patients presenting to outpatients and emergency department of obstetrics and Gynecology, in RL Jalapa Hospital and Research Centre affiliated to Sri Deva Raj Urs Academy of Higher Education and Research, Kolar between December 2020 and February 2021, a study conducted among women with a history of unsupervised pills abortion pill intake.

Results: In the study $35 \%$ were in the age group 19 to 20 years, $45 \%$ were in the age group 21 to 25 years and $20 \%$ were in the age group 26 to 30 years. 55\% were from joint family and $45 \%$ were from nuclear family, $76.2 \%$ were multigravida and $23.8 \%$ were primigravida.

Conclusions: This study shows strict legislation and restriction required the sale of abortion pills for public use. The drug should be available only via health care facilities under the super version during an abortion. Creating awareness regarding contraception and effective method to fulfil the unmet need for contraception will be useful avoid the harmful practice of self-administered abortion pills and reducing maternal mortality.

Keywords: Pills, Abortion, MTP, Contraception

\section{INTRODUCTION}

In India, it is becoming a public health problem due to easy over-the-counter abortion pill availability despite the legal ban, widespread misuse by non-allopath doctors, dais and quacks, and ignorance on part of women. The pills are being dispensed blindly without proper medical evaluation and even without ruling out the contraindications. In India, abortion facility is available legally under the MTP act, 1971. Despite this, women who want to terminate a pregnancy often ignore the legal status of abortions and have unsafe abortions. ${ }^{1}$
The WHO defines unsafe abortion as a procedure for terminating an unwanted pregnancy either by persons lacking the necessary skills or in an environment lacking minimal medical standards or both. ${ }^{2}$

The guidelines for medical abortion in India have been prepared by WHO in human reproduction, All India Institute of Medical Sciences, in collaboration with the Ministry of Health and Family Welfare, Government of India, and Indian Council of Medical Research according to which medical abortion is approved up to seven weeks of pregnancy. ${ }^{3}$ 
Medical abortion with mifepristone and misoprostol is a very safe option for termination of pregnancy when consumed under medical supervision with a success rate of $92-97 \% .^{4}$ It also emphasizes the need to obtain a detailed medical history to rule out contraindications for medical abortion such as bleeding disorders, uncontrolled seizure disorder, chronic adrenal failure, etc, and the importance of bimanual examination to assess the size of the uterus and a basic laboratory workup.

\section{Objectives}

The objectives of this study were to determine the complications and consequences including maternal morbidity and mortality following indiscriminate selfconsumption of abortion pills reporting to a tertiary care center.

\section{METHODS}

Its cross-section study including 81 patients presenting to outpatients and emergency department of obstetrics and Gynecology, in RL Jalapa Hospital and Research Centre affiliated to Sri Deva Raj Urs Academy of Higher Education and Research, Kolar between December 2020 and February 2021, a study conducted among women with a history of unsupervised pills abortion pill intake.Unsupervised intake implies when a pill is procured without authorized medical prescription and or pill has been prescribed by untrained personnel like dais and quacks and or consumed without the following protocol as laid down by the Medical termination of pregnancy act of India (MTP act).
A detailed history gathered, age, parity, education status, last menstrual period, presenting complaints, source of pills reason of abortion.

All diagnostic modalities were completed including complete clinical examination, investigation $\mathrm{Hb}$ level on admission, whether a patient was in shock, USG finding of an incomplete or complete or failed abortion, evidence of sepsis-like fever and tenderness on pelvic examination, blood transfusion, treatment is given duration and of hospital stay. Management was based on whether the patient was bleeding profusely when the surgical evacuation was performed whereas when bleeding was less and the amount of retained products as assessed by ultrasounds was minimal medical methods were used. Data were entered into a Microsoft excel datasheet and were analyzed using SPSS 22 version software. Categorical data was represented in the form of Frequencies and proportions.

\section{RESULTS}

The total number of abortions in our institution including spontaneous and induced abortions between the periods of December 2020-January 2021 was 81 . In the study $35 \%$ were in the age group 19 to 20 years, $45 \%$ were in the age group 21 to 25 years and $20 \%$ were in the age group 26 to 30 years. $55 \%$ were from joint family and $45 \%$ were from nuclear family, $76.2 \%$ were multigravida and $23.8 \%$ were primigravida..$^{5} 10 \%$ were in the gestational age $<12$ weeks, $41.2 \%$ were in the gestational age 12 to 20 weeks and $48.8 \%$ were in the gestational age 21 to 24 weeks (Table 1).

Table 1: Profile of subjects in the study.

\begin{tabular}{|lll|l|}
\hline Variables & & Count & Percentage (\%) \\
\hline \multirow{3}{*}{ Age (years) } & 19 to 20 & 28 & 35.0 \\
\hline \multirow{2}{*}{ Type of family } & 21 to 25 & 36 & 45.0 \\
\cline { 2 - 4 } & 26 to 30 & 16 & 20.0 \\
\hline \multirow{2}{*}{ Parity } & Joint & 44 & 55.0 \\
\hline \multirow{3}{*}{ Gestation age (weeks) } & Nuclear & 36 & 45.0 \\
\hline & Primigravida & 19 & 23.8 \\
\cline { 2 - 4 } & Multigravida & 61 & 76.2 \\
\hline & $<12$ & 8 & 10.0 \\
\hline & 12 to 20 & 33 & 41.2 \\
\cline { 2 - 4 } & 21 to 24 & 39 & 48.8 \\
\hline
\end{tabular}

Source of pills procurement majority $67 \%$ patient procured pills by husband, $4 \%$ by allopath, and $29 \%$ by self (Figure 1 ). No of days since consumption of pills to hospital visit 1 day in $3.8 \%, 2$ days in $38.8 \%, 3$ days in $55 \%$ and 6 days in $2.5 \%$ shown in Figure 2. Pregnancy was confirmed by 39 women with a urine pregnancy test and 22\% USG was done prior to pill consumption along with UPT shown in Figure 3.

One patient underwent $\mathrm{D}$ and $\mathrm{C}$ elsewhere, but still complain of irregular bleeding and was diagnosed to have an incomplete abortion. Finding that $65 \%$ had heavy bleeding, $36.2 \%$ had a mass per vagina, $27.5 \%$ had irregular bleeding, $6.2 \%$ had a shock, $47.5 \%$ had pain abdomen, $65 \%$ had an incomplete abortion, 5\% had a fever, $8.8 \%$ had vaginal discharge, $18.8 \%$ had missed abortion, $13.8 \%$ had ectopic pregnancy, $3.8 \%$ had hydatiform mole, $11.2 \%$ had sepsis, $1.2 \%$ had scar pregnancy and $2.5 \%$ had maternal death shown in Figure $4 .^{6}$ In the study $80 \%$ received medical treatment, in $10 \%$ shock was managed, $11.2 \%$ received IV oxytocin, $26.2 \%$ received misoprostol tablet, $100 \%$ received IV antibiotics, 
$10 \%$ had suction and evacuation, $7.5 \%$ were given oral iron, $13.8 \%$ underwent exploratory laparotomy listen in the shown in Figure 5.

The prevalence of anemia in pregnant women in our country is almost $87 \%$ and the estimated number of maternal deaths due to anemia is $22,000 /$ year. ${ }^{7}$ Selfmedication of abortion pills in women with severe anemia could be fatal. In the study $41.2 \%$ did not receive blood transfusion, $23.8 \%$ received 1 blood transfusion, $30 \%$ received 2 blood transfusion, $3.8 \%$ received 3 blood transfusion and $1.2 \%$ received 4 blood transfusion shown in Figure $6 .^{8}$

Patients who presented with profuse bleeding were managed by immediate surgical evacuation whereas when bleeding was minimal and with the USG showing minimal products of conception vaginal misoprostol was used to complete the procedure.

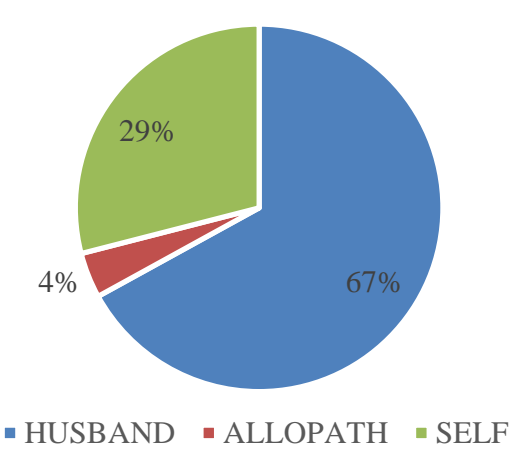

Figure 1: Source of pills procurement.

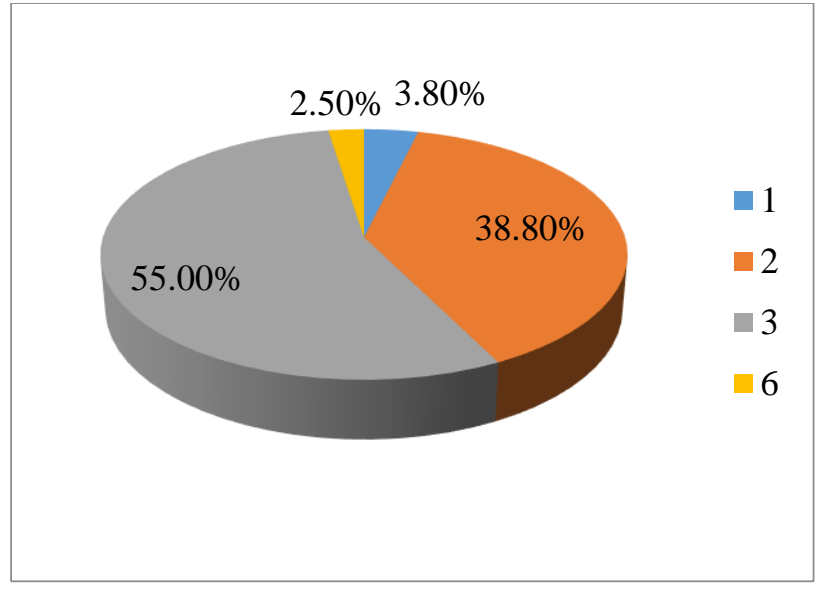

Figure 2: Time interval between pill intake at visit to a hospital in days distribution.

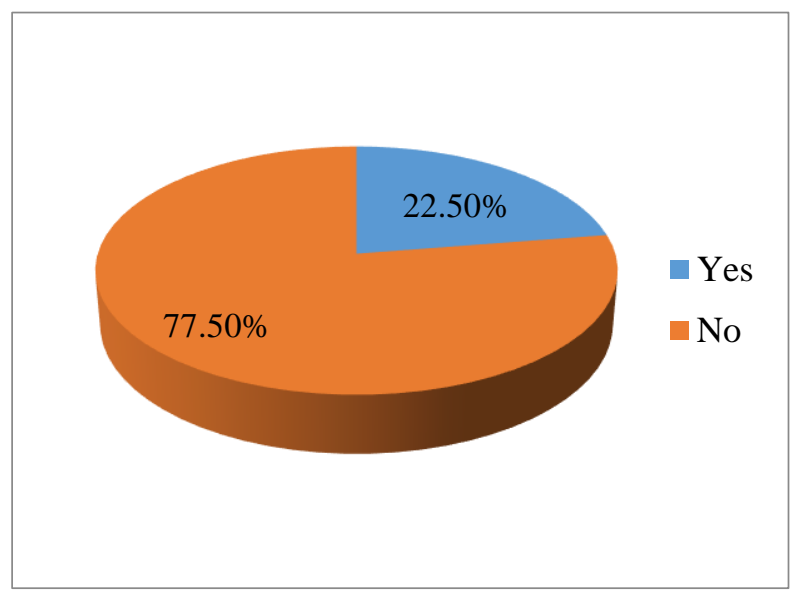

Figure 3: USG done before consumption of the pill.

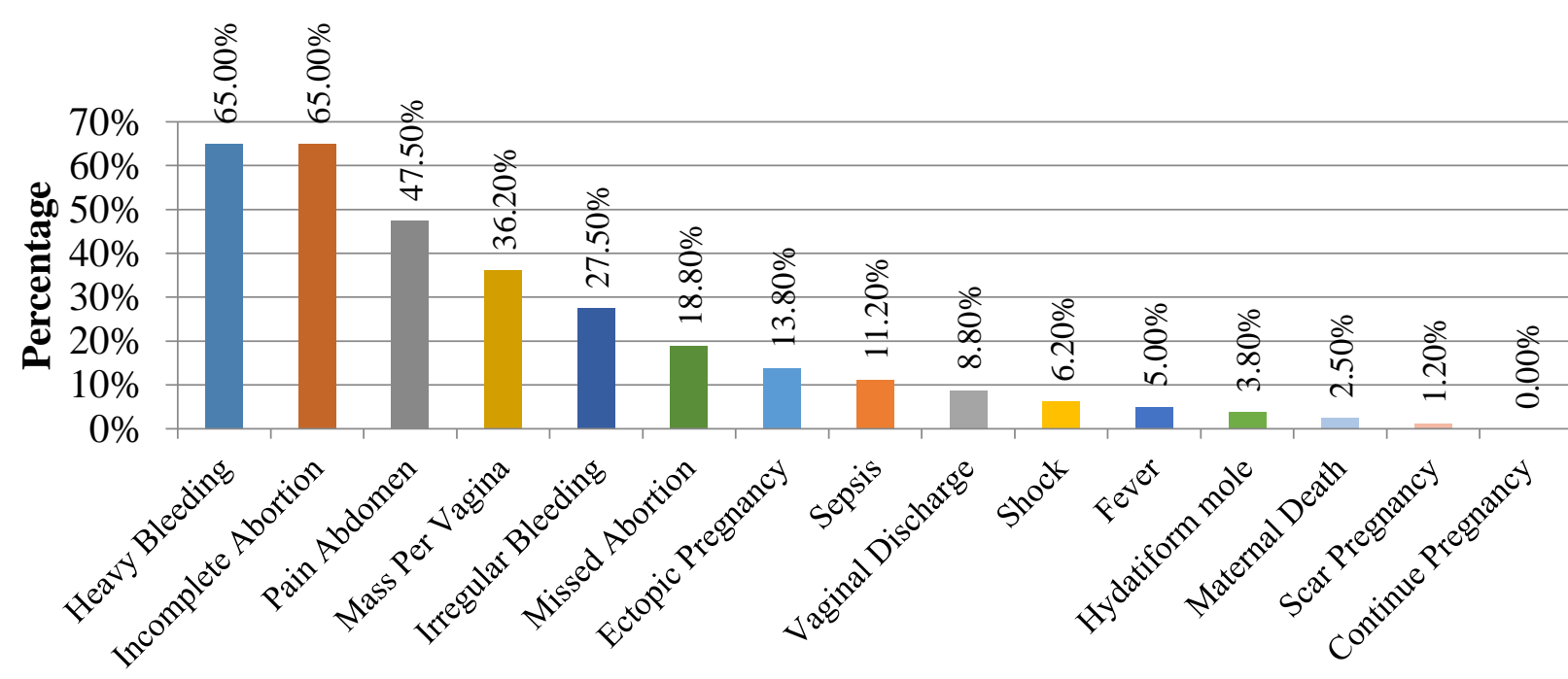

Outcome

Figure 4: Outcome of pill usage. 


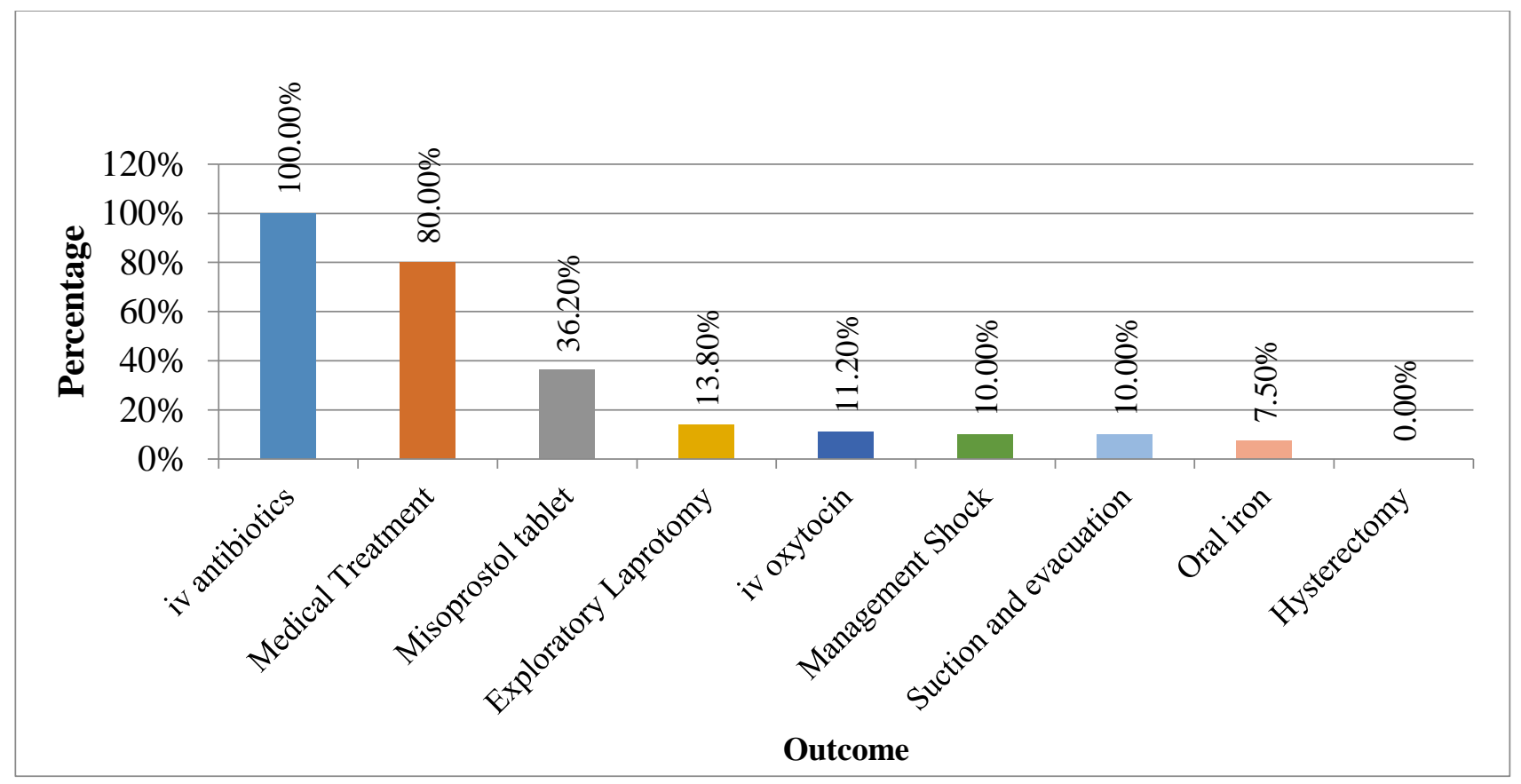

Figure 5: The treatment given.

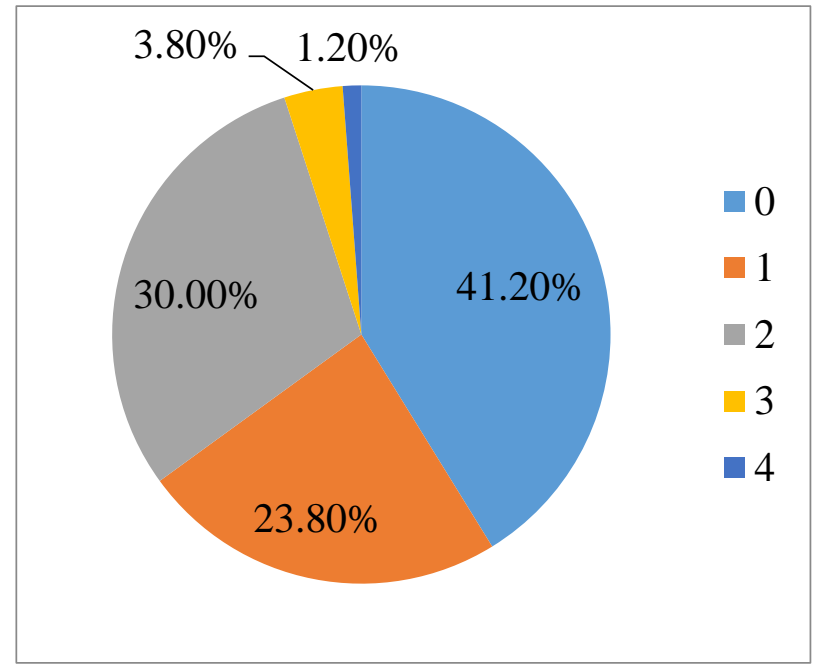

Figure 6: Blood transfusion distribution.

\section{DISCUSSION}

The MTP act of India, legalizing abortions was passed to reduce the number of maternal deaths due to unsafe abortions, but still, maternal deaths are attributed to unsafe abortions in India.

We found that almost $75 \%$ of patient with incomplete abortion in our hospital, during study came history of selfadministered with abortion pills despite clear guidelines that these pills have to be taken under supervision and to be prescribed by authorized under the MTP act. This is due to the easy availability of pills in rural areas.

The prevalence of anemia in women in our country is almost $87 \%$ and the estimated number of maternal deaths due to anemia is 22,000 years. Self-medical abortion pills in women with severe anemia could be fatal. ${ }^{9}$ In our study, self-administered pills among low social-economic status women seen Owing to their ignorance and unwariness of compilation associated it like shock, hemorrhage, sepsis, maternal deaths. Most of the women $84 \%$ were illiterate and formal education only at the primary level, been influenced by untrained and unauthorized pill providers like Dais, Quarks. Unintended pregnancy, failure of contraception, or due to socio-economic condition without medical consultation despite the availability of various methods of contraception. The majority of the women were not aware of the contraceptive options available at health centers, hence, accounting for high (48\%) contraceptive non-users in our study, hence points to the high unmet need for contraception. This scenario is alarming as the women are not using proper contraceptive methods despite the various national programs propagating their use. ${ }^{10}$

Common infection after any medical abortion is seen is Clostridium dorselli infection, mostly seen with unsafe abortion and associated various symptoms like fever, vomiting, foul-smelling discharge. The scar rupture in cesarean pregnancy was seen in the second trimester of medical abortion but risk rupture was also seen of around $1.2 \%$. We had $4(10 \%)$ patients with post-cesarean pregnancy in our study who had consumed abortion pills even without confirmation of gestational age with the existent risk of scar rupture which could have been fatal. $65 \%$ of patients had profuse bleeding managed by immediate surgical evacuation, bleeding was minimal with USG showing minimal products of conception, misoprostol vaginal was used complete the procedure. 
Medical disorders like epilepsy, cardiac disease, and bronchial asthma were seen in 4 patients that make highly risky. None of found to have a Rhesus negative group. Mostly medical abortion, we find that our society will go for medical abortion as a safe option for terminating a pregnancy, the reason behind lack of awareness of the complication involved in medical abortion, the stay of the hospital, the cost involved, and mainly because of easy availability drug without any restriction. Second-trimester medical abortions done in this manner could be also sexselective in our scenario. At the same time, overlook the fact that unsupervised self-administration of abortion pills turns out to be disastrous and will lead to shock, severe anemia, sepsis, and ectopic pregnancies. These unnecessary visits are due to a lack of pre-abortive counseling. Most of the patients did not take pills as per protocols. Again, we should not forget that majority of these patients did not take pills as per recommended protocol. The MTP act of India, legalizing abortions was passed to reduce the number of maternal deaths due to unsafe abortions. But still of maternal deaths are attributed to unsafe abortions in India. Any procedure which is performed outside the bounds of law tends to be unsafe and 5 million unsafe abortions are performed per year in India. $^{11}$

At the same time, overlook the fact that unsupervised selfadministration of abortion pills turns out to be disastrous and will lead to shock, severe anemia, sepsis, and ectopic pregnancies. These unnecessary visits are due to a lack of abortive counselling. Most of the patients did not take pills as per protocols. Again, we should not forget that majority of these patients did not take pills as per recommended protocol.

\section{CONCLUSION}

This study shows strict legislation and restriction required the sale of abortion pills for public use. The drug should be available only via health care facilities under the super version during an abortion. Creating awareness regarding contraception and effective method to fulfil the unmet need for contraception will be useful avoid the harmful practice of self-administered abortion pills and reducing maternal mortality. An awareness program should be made to make chemists and the public awareness of the MTP act and its consequences. Training and up-to-date on various methods will help in improving their knowledge from time to time.
Funding: No funding sources

Conflict of interest: None declared

Ethical approval: The study was approved by the Institutional Ethics Committee

\section{REFERENCES}

1. WHO. International Consensus Conference on Nonsurgical (Medical) Abortion in Early First Trimester on Issues Related to Regimens and Service Delivery. Frequently asked clinical questions about medical abortion. Geneva: WHO; 2006.

2. Mishra N. Unprecedented use of medical abortion can be injurious to health. JEMS. 2013;2(8):856-9.

3. Guidelines for medical abortion in India, 2021. Available at: http://ebookbrowsee.net/guidelines formedicalabortion-in-india-doc-d134818684. Accessed on 01 June 2021.

4. Desalegn S. Prevalence of anaemia in pregnancy in Jima town, South-western Ethiopia. Ethiop Med J. 1993;31(4):251-8.

5. Clark W, Bracken H, Tanenhaus J, Schweikert S, Lichtenberg ES, Winikoff B. Alternatives to a routine follow-up visit for early medical abortion. Obstet Gynecol 2010;115:264-72.

6. Dakhale GN, Hiware SK, Shinde AT, Mahatme MS. Basic biostatistics for post-graduate students. Indian J Pharmacol. 2012;44(4):435-42.

7. Ellertson $\mathrm{C}$, Waldman $\mathrm{SN}$. The mifepristonemisoprostol regimen for early medical abortion. Curr Womens Health Rep. 2001;1(3):184-90.

8. Rao SPSS. Richard J. An Introduction to Biostatistics, A manual for students in health sciences. 4th ed. New Delhi: Prentice-Hall of India; 2006: 86-160.

9. Elenbaas RM, Elenbaas JK, Cuddy PG. Evaluating the medical literature. Part II: Statistical analysis. Ann Emerg Med. 1983;12(10):610-20.

10. Silva WI, Dayananda RA, Nishanthi PNW. Contraceptive behavior of abortion seekers in Sri Lanka. Asian Popul Stud. 2006;2(1):3-18.

11. Thaker RV, Deliwala KJ, Shah PT. Self-medication of abortion pill: women's health in Jeopardy. NHLJMS. 2014;3(1):26-31.

Cite this article as: Singh S, Rangappa SS. Selfadministered medical abortion pills: evaluation of the clinical outcome and complications among women presenting with unsupervised pill intake to a tertiary care hospital: a cross-sectional study. Int J Reprod Contracept Obstet Gynecol 2021;10:3424-8. 